\title{
Editorial
}

\section{L'ESPRIT D'ENTREPRISE DES UNIVERSITÉS ET LA CONCURRENCE : LE CAS DES PETITES UNIVERSITÉS CANADIENNES $\dagger$}

\author{
CHARLES H. BÉLANGER*
}

\section{RÉSUMÉ}

Cet article traite de la recherche dans les petites universités $(<5000$ étudiants à plein temps) et de la situation assez particulière qui les caractérise à cet égard. Ces établissements, que l'on pense généralement axés sur l'enseignement dans les arts libéraux, se consacrent avec succès à la formation de scientifiques. Les suggestions pratiques de planification stratégique présentées ici visent à augmenter leur pouvoir compétitif sur la scène universitaire et à leur éviter de sombrer dans l'oubli.

A l'image du pays, la cinquantaine d'universités canadiennes présente au public une mosaïque remplie de contrastes et d'énigmes. Financées à plus de $80 \%$ par les deniers publics, elles jouissent d'une autonomie inégalée dans les autres secteurs. Leurs professeurs bénéficient d'un degré d'indépendance qui fait l'envie des

\footnotetext{
*Vice-recteur à l'enseignement et à la recherche/Vice President Academic

Professeur d'informatique de gestion/Professor of management information systems Université Laurentienne/Laurentian University
}

$\dagger$ Article accepté pour publication dans la revue Politiques et Management Public 7 (3) 1989, et publié ici avec l'autorisation des éditeurs. 
personnes astreintes à des horaires rigides de travail. Le fait que toutes les universités sans exception se donnent trois fonctions (l'enseignement, la recherche et le service à la collectivité) mais que l'enseignement soit largement perçu par le public comme l'unique fonction contribue à l'ambiguïté de leur rôle et de celui du professeur. Le mode collégial de gestion des universités leur permet de profiter d'une étonnante richesse intellectuelle mais rend aussi les consciences individuelles vulnérables en regard des objectifs, des priorités et de la planification institutionnelle.

\section{LE CONTEXTE}

A peu près la moitié des universités canadiennes compte moins de 5000 étudiants à plein temps. On associe souvent ces établissements à des universités régionales, axées principalement sur l'enseignement de premier cycle et, plus souvent qu'autrement, entraînées par le courant des universités de taille supérieure qui, elles, sont décrites comme des universités de grands centres urbains axées davantage sur la recherche. Même s'il ne s'agit que d'une question d'accent plutôt que de monopole, cette caractéristique importante rend souvent la co-existence entre ces deux catégories d'universités semblable au partage d'un même pays par deux groupes de cultures différentes. De part et d'autre, il y a un manque d'appréciation des forces et des faiblesses de l'autre groupe, ce qui a tendance à rendre la discussion plus émotive que rationnelle. Les petites universités se disent victimes du réseau des grandes universités quand vient le temps des octrois de fonds de recherche, tandis que les grandes universités avouent à demi-mots qu'elles doivent établir des normes parallèles pour répondre aux demandes des petites universités. Cependant, les données n'appuient pas toujours ces accusations. Les rapports du Conseil de recherches en sciences humaines montrent que les petites universités reçoivent des subventions dans une mesure légèrement supérieure à la proportion que représente leur nombre de chercheurs. En ce qui a trait au Conseil de recherches en sciences naturelles et en génie, les petites universités accusent du retard à la fois sur le taux de succès et les sur montants reçus par chercheur. Quant à la question des normes parallèles, il est intéressant de relater les résultats d'une étude américaine intitulée "The Future of Science at Liberal Arts Colleges" (Oberlin College, 1985). En général, les données montrent que les 48 principaux collèges d'arts libéraux se classent en tête, ou peu s'en faut, de tous les établissements américains d'enseignement supérieur pour la formation des scientifiques (y compris les multi-universités et les principaux centres de recherches). Ces données montrent que les collèges tirent la majeure partie de leur succès des relations étroites entre les professeurs et les étudiants. Ce même rapport indique que les principaux collèges d'arts libéraux ont non seulement un rendement exceptionnel dans les sciences naturelles mais qu'ils fonctionnent aussi à moindre coût. Bien qu'une pareille étude n'ait jamais été menée au Canada, le parallèle entre les "liberal arts colleges" américains et les petites universités canadiennes est assez frappant. 
La concurrence. Aujourd'hui, toutes les universités, peu importe leur taille, se font concurrence. Cette situation a des conséquences bénéfiques pour certaines et néfastes pour d'autres, selon les orientations choisies et les leaders ou stratèges en place pour poursuivre ces axes d'excellence. Depuis au moins deux décennies, on ne cesse de nous dire que le secteur postsecondaire est devenu une "grosse entreprise" à la manière du secteur privé. Il est évident que dans le privé, si l'entreprise ne peut pas faire face à la compétition, elle doit inévitablement fermer ses portes. Dans son livre intitulé Academic Strategy, Keller (1983) mentionne que d'ici 1995 , entre 10 et $30 \%$ des 3100 universités et collèges américains devront fermer leurs portes à cause d'un manque d'étudiants et de ressources. Toutes nos universités canadiennes étant financées par le gouvernement, il faut quasiment exclure la possibilité de fermeture de certaines d'entre elles par celui-ci. Dans ce contexte, la concurrence entre établissements n'est qu'imaginaire car ils finissent toujours par demander au gouvernement de les sortir de l'impasse. Cet état de chose produira cependant une catégorie d'universités languissantes. Que peuvent faire les petites universités pour éviter de se retrouver dans cette catégorie? Écoutons les conseils des experts Keller (1983) et Sibley (1986):

1. L'établissement doit se doter d'un président ou recteur possédant des qualités éprouvées de stratège et de chef de file. Si l'on en croit les conclusions de Presidents Make a Difference (Kerr, 1984), il est de plus en plus difficile d'attirer des recteurs d'universités qui répondent à ces exigences car ils hésitent à affronter les lourds mécanismes décisionnels et les imprévus que leur réserve ce milieu en constante effervescence.

2. Nos entreprises universitaires devront se poser beaucoup plus de questions et s'évaluer plus rigoureusement en ce qui touche le management du changement. Dans quelles sortes d'affaires sommes-nous vraiment engagés? Quel rôle spécial jouons-nous? Quels sont nos avantages par rapport à des établissements similaires? Quels objectifs cette université devrait-elle avoir atteints d'ici dix ans? Quelles sont nos traditions, nos valeurs, nos aspirations? Comment relions-nous ces traditions, les valeurs et les aspirations à un monde extérieur continuellement en évolution? Quels sont nos points forts et nos faiblesses? Dans quelle partie du marché avons-nous l'assurance d'affronter efficacement la concurrence? De quelle façon nous distinguerons-nous des autres joueurs de la ligue?

3. La notion de collégialité devra se transformer de deux façons. Premièrement, il sera nécessaire d'établir un esprit de coopération entre administrateurs et professeurs afin de mieux promouvoir l'entreprise. Deuxièmement, il faudra reconnaître la nécessité de maintenir la collégialité (nous entendons par là le contrôle collectif, par un groupe de pairs, de questions relevant de leur compétence professionnelle) mais il faudra aussi restreindre la responsabilité de ce contrôle collectif sur tout ce qui se passe dans l'établissement.

4. Le concept de qualité doit passer du stade de l'imaginaire, dans plusieurs cas, à une réalité qui se traduit par une plus grande satisfaction du consommateur- 
étudiant et par un apport concret au milieu. Il en découlera forcément une spécificité et une différence plus marquées entre les établissements.

L'esprit d'entreprise du corps professoral. Ayant généralement défini le contexte de la concurrence entre les universités, nous pouvons maintenant aborder la question de l'esprit d'entreprise du corps professoral. Si étonnant que cela puisse paraître à certains, disait Lacroix (1988), il y a une belle analogie à faire entre l'entrepreneur du monde des affaires et le professeur d'université. "Tous deux ont un degré de liberté très grand pour combiner les ressources à leur disposition; tous deux sont essentiellement jugés à leur "output". Tous deux doivent être innovateurs pour réussir. Tous deux sont pleinement responsables de leurs succès et de leurs échecs. Ils ne sont pas des exécutants, etc." Chaque professeur est essentiellement employé et chef d'entreprise. Il est un employé dans sa fonction d'enseignement puisqu'il doit se conformer à un certain nombre d'heures, à des horaires fixes et faire face à toute une série de responsabilités envers ses étudiants et son département. Il est aussi un chef d'entreprise dans la conduite de ses activités de recherche.

Le fait que la fonction de recherche soit une obligation de tout professeur d'université mais que la conduite de cette recherche soit entièrement laissée au dynamisme et à l'initiative de chaque professeur donne lieu à des performances extrêmement variables. Une étude (Bélanger et Lacroix, 1988) menée à l'Université de Montréal, université dite de recherche, a révélé (tableau 1) que $40 \%$ des professeurs formant le groupe le plus productif de l'université avaient à leur actif :

- 91,1\% des publications hautement spécialisées

- $73,7 \%$ d'autres productions externes

- $63,6 \%$ des thèses de maîtrise et de doctorat complétées

- 65,4\% des subventions et de certains contrats de recherche

- 12 crédits d'enseignement par rapport à 12,4 pour l'ensemble du corps professoral.

Ces résultats prouvent que l'esprit d'entreprise est très développé chez certains et plutôt absent chez d'autres. Jusqu'à quel point peut-on généraliser ces conclusions? Dans la mesure où la productivité de recherche de l'Université de Montréal la place au cinquième rang parmi les établissements de grande taille au Canada (Bélanger et Lacroix, 1986), et étant donné qu'une pareille étude n'a pas été effectuée auprès des petites universités, il n'est pas utopique de penser que les $40 \%$ de professeurs les plus performants dans les petites universités auraient des résultats semblables. Cependant, même si cette analogie se révélait exacte, elle offre très peu de réconfort. 
Contribution des $40 \%$ de professeurs les plus productifs selon le secteur disciplinaire

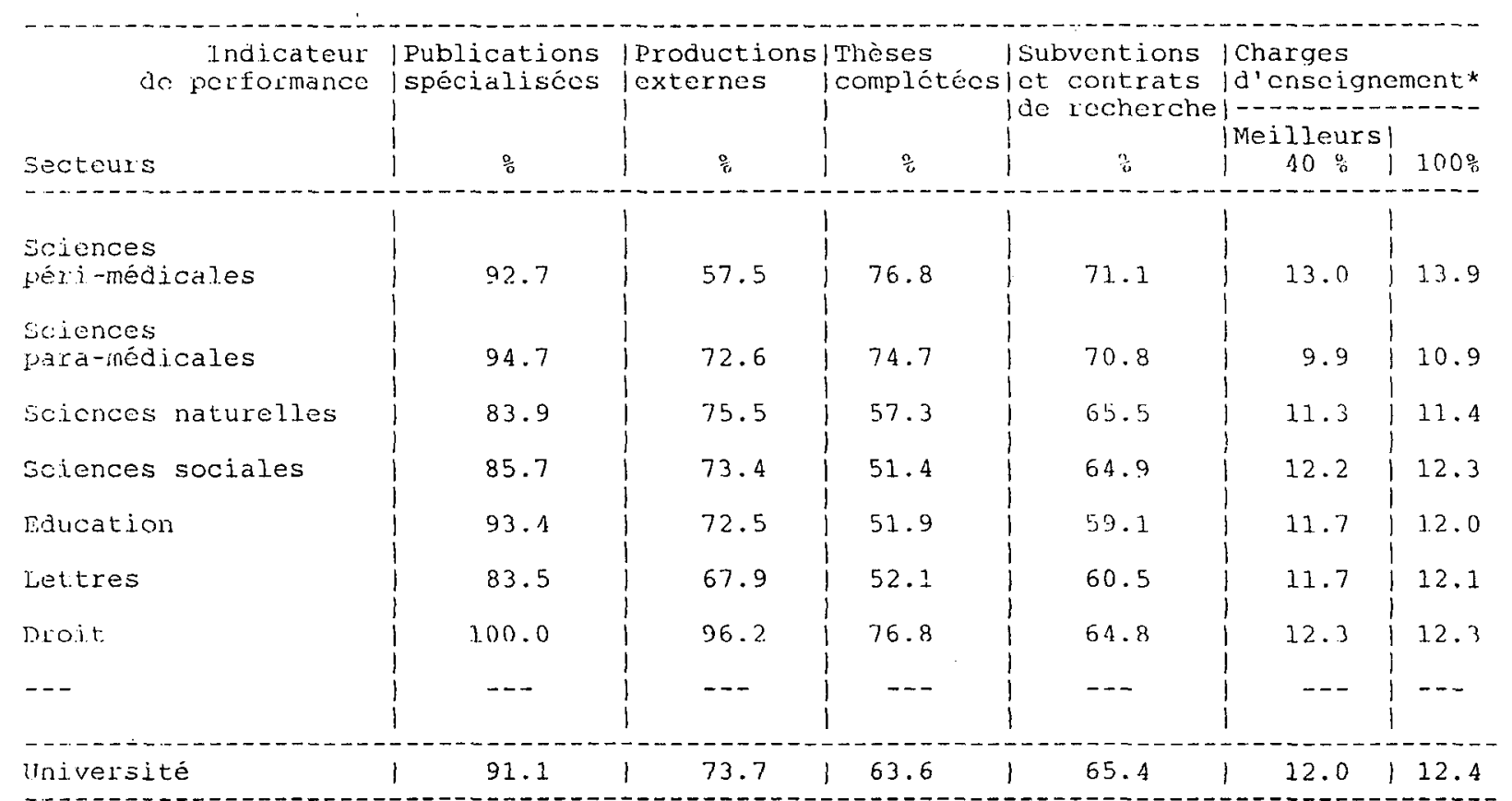

* Les charges d'enseignement tiennent compte des dégrèvements pour responsabilités administratives. 


\section{LES CONTRAINTES}

A mesure que la compétition pour les subventions de recherche distribuées par les trois grands conseils subventionnaires fédéraux s'intensifie, les petites universités se rendent compte de l'inégalité des forces, surtout lorsqu'il s'agit d'un même territoire convoité par des équipes de recherche établies. En ce qui a trait aux subventions destinées à des chercheurs individuels, la problématique se présente différemment. C'est justement l'intensité de cette concurrence qui a incité les petites universités à organiser, depuis 1985, une conférence annuelle sur la recherche afin de discuter de leurs problèmes et d'envisager certaines stratégies. Il semble qu'après la publication de trois rapports importants sur ces questions, au milieu des années 70 (Overgaard, 1974; Isaacs, 1976; Conseil des arts, 1977), il y a eu une diminution d'intérêt durant les années suivantes. Cependant, il ne faut pas s'étonner outre mesure de constater que les contraintes qui freinaient le climat de recherche dans les petites universités il y a dix ans n'ont pas énormément changé aujourd'hui. Passons brièvement en revue ces principales contraintes, non pas pour le plaisir de formuler des excuses, mais plutôt pour mieux connaitre les limites des petites universités et articuler un plan d'attaque comme le ferait le chef d'une petite entreprise.

1. Les charges d'enseignement. Une étude menée par Bélanger, Lacroix et Raynauld (1987) auprès de 39 universités canadiennes démontre qu'effectivement les charges d'enseignement dans les petites universités hors Québec se situent plutôt autour de 17-17,5 crédits ${ }^{1}$ par année par rapport à 13-13,5 crédits dans les universités de grande taille, soit une différence de près de $25 \%$. La même étude a révélé que le corps professoral distribuait son énergie dans des proportions bien différentes entre l'enseignement et la recherche dans les deux catégories d'universités (tableau 2). De façon générale, plus l'établissement est petit, plus les charges d'enseignement sont élevées et plus les groupes d'étudiants sont petits, ce qui constitue un argument de la défense des grandes universités.

2. Le manque de programmes d'études supérieures. Il est indiscutable que les petites universités ont toujours eu plus de difficultés à créer et à soutenir une masse critique de programmes d'études supérieures. Les petites universités avancent à juste titre qu'elles ne bénéficient pas des services d'étudiants des études avancées comme aide à l'enseignement et à la recherche. Cependant, les grandes universités avancent l'argument contraire, soit que l'encadrement des étudiants de maîtrise et de doctorat exige une présence et une attention sans commune mesure avec l'étudiant de premier cycle. Alors que la proportion d'étudiants de niveau supérieur par professeur est inférieure à 0,5 dans les petites universités, elle se situe autour de 2,0 étudiants par professeur dans les grandes universités (Bélanger, Lacroix et Raynauld, 1987).

3. La taille des unités. Ce facteur semble être un des plus importants à examiner. Des études américaines ont conclu que le nombre critique de professeurs dans

${ }^{1}$ Unités de valeur (U.V.) en France. 
TABLEAU 2

Distribution du temps de travail du corps professoral

\begin{tabular}{|c|c|c|c|}
\hline & $\begin{array}{l}\text { Petites } \\
\text { universités }\end{array}$ & $\begin{array}{l}\text { Grandes } \\
\text { universités }\end{array}$ & $\begin{array}{l}\text { Moyenne } \\
\text { pondérée }\end{array}$ \\
\hline Eonctions & $(N=16)$ & $(\mathrm{N}=14)$ & $(N=31)^{*}$ \\
\hline Enseignement & $54 \%$ & $48 \%$ & $52 \%$ \\
\hline Recherche & $26 \%$ & $33 \%$ & $28 \%$ \\
\hline Service & $20 \div$ & $19 \%$ & $20 \%$ \\
\hline
\end{tabular}

* Le total 31 exclut les 8 universités répondantes du Québec

un département peut varier selon la discipline mais que dans l'ensemble, il est difficile d'aller en-deça d'une dizaine. Le fait est que dans les petites universités, la plupart des unités disciplinaires se situent en-deçà du nombre critique et que des effets plutôt négatifs pour la recherche en découlent. Un professeur de petite université doit préparer une plus grande variété de cours. II est quasi impossible de former des équipes disciplinaires de recherche; par exemple, alors qu'une petite université peut compter sur les services d'un spécialiste en chimie analytique, il n'est pas rare d'en compter 6-8 dans les départements des grandes universités. Les petites universités présentent le danger inhérent de l'isolement disciplinaire sans parler de l'éloignement géographique d'avec les collègues d'autres établissements. Les conflits de personnalité et d'idées semblent être plus prononcés et personnels. Il est beaucoup plus difficile de "camoufler" un ou deux collègues dans une équipe de cinq ou six que d'en camoufler huit ou neuf dans une équipe de 35 quand vient le temps de passer le test de l'évaluation pour l'obtention ou la reconduction d'un programme de deuxième ou troisième cycle. Finalement, il y a souvent un manque de concurrence et d'émulation entre collègues dans les petites unités.

4. La pénurie de "modèles". Il existe un dicton en management qui dit : "If you want to go places, you associate with movers". Le même dicton s'applique à la recherche. Les jeunes professeurs ont besoin de la présence de professeurschercheurs chevronnés et même de s'y associer pour apprendre les rouages de l'entreprise privée que constitue la fonction recherche. Les organisations privées de qualité et certains départements de prestige d'universités remplis- 
sent ce rôle d'émulation à merveille. Les petites universités ont des chercheurs qui peuvent jouer ce rôle et le font, mais elles en ont relativement peu. D'abord, il est plus difficile de les attirer en raison des charges d'enseignement plus élevées et du petit nombre de programmes des cycles supérieurs. De plus, ces professeurs sont déjà trop sollicités en raison de leur excellence générale et ils en arrivent à ralentir leur carrière de recherche au cours des années parce qu'ils ont dû accomplir des tâches que personne d'autre ne voulait ou ne pouvait assumer.

5. Infrastructure et ressources inadéquates. Il est évident que la notion d'infrastructure et de ressources est présente à l'esprit de tous les chercheurs aussi bien dans les petites que dans les grandes universités. Cette notion revêt cependant une importance et un caractère différents selon que vous appartenez à une discipline nécessitant des laboratoires, telle la chimie plutôt qu'à une discipline ne nécessitant que l'usage de crayons et de livres, telle la littérature. Lorsque l'on considère les trois grands conseils fédéraux distributeurs de subventions, on constate que la subvention moyenne par professeur varie entre 14000 \$ et $17000 \$$ dans les universités de recherche alors qu'elle se situe entre 3000 \$ et $10000 \$$ dans les universités d'enseignement, selon que l'établissement offre des programmes de doctorat, et particulièrement des programmes en sciences de la santé auxquels le Conseil de recherches médicales du Canada peut allouer des fonds. Cette différence importante entre les deux catégories d'universités ne règle certainement pas la carence d'équipement, d'infrastructure et de ressources humaines de soutien à la recherche dans les petites universités.

6. Parti-pris contre les petites universités. Au point de départ, les petites universités se retrouvent dans une situation défavorable avant même de faire face à la concurrence, en raison de leur contexte historique, de leur accent sur la relation étudiant-professeur, de leur isolement relatif des grands centres urbains et du fait qu'elles sont tout simplement de petite taille. Elles forment donc un réseau de professeurs moins étendu et moins présent et un réseau de diplômés qui se limitent à certaines disciplines et professions, pas toujours les plus représentées dans nos mécanismes de décision.

\section{PLANIFICATION STRATÉGIQUE}

Si la liste de toutes ces contraintes vous décourage, je vous suggère de quitter le monde des petites universités, car la recherche ne peut pas être séparée de l'enseignement sans éventuellement constituer un poids mort pour les étudiants et tout l'établissement. Si par ailleurs, vous êtes compétitif et combatif, ce qui est une qualité sine qua non de tout chef d'entreprise, examinons comment on peut contourner ces contraintes.

Première étape. Premièrement, il faut retourner au premier point mentionné dans le cadre de la planification stratégique. L'établissement doit se doter d'un 
recteur, d'un stratège qui comprend non seulement l'idée de l'entreprise universitaire en général, mais aussi celle de son université. Cette pièce de l'échiquier est tellement fondamentale que sa présence engendre toute une séquence de décisions qui assureront la vitalité à l'établissement. N'est-ce pas ce que fait également l'entreprise privée? Ce nouveau stratège s'entoure généralement d'une équipe dynamique qui partage sa philosophie de cet établissement.

Deuxièmement, l'administration -jusqu'au directeur de département- doit comprendre et expliquer que les politiques d'embauche, de promotion et de permanence forment essentiellement les grands jalons d'une politique de recherche. Si l'établissement embauche et retient des personnes qui n'ont ni l'aptitude ni la volonté de devenir des chefs de petite entreprise, la frustration surpassera l'esprit d'entreprise potentiel.

En troisième lieu, se pose la question des quelques axes de développement de recherche que l'établissement devra favoriser. Bien que difficiles, ces choix sont essentiels pour l'économie de ressources et la création de masse critique entre disciplines. Autant la recherche disciplinaire d'équipe est le château fort des grandes universités, en particulier en ce qui a trait au Conseil de recherches en sciences naturelles et génie et au Conseil de recherches médicales, autant les petites universités doivent faire de la recherche interdisciplinaire leur marque de fabrique et leur cheval de bataille. L'organisation de la recherche autour de quelques axes ou centres institutionnels a également des retombées positives pour les professeurs-chercheurs indépendants. Cette structure leur permet de se concentrer sur un ensemble de priorités institutionnelles et leur procure un foyer d'échanges avec les autres chercheurs ayant les mêmes visées; de plus, ils ont.la liberté de poursuivre leurs intérêts personnels de recherche s'ils le désirent.

Deuxième étape. Disons tout de suite que cette deuxième étape ne sera utile que si les trois points de la première étape sont bien identifiables. Il s'agit ici d'outils, de moyens et de politiques que l'établissement utilise pour favoriser le climat de recherche et appuyer les efforts des chercheurs. En premier lieu, il semble qu'un bureau de la recherche efficace remplit un rôle essentiel. Dans son rapport au Conseil des sciences du Canada, Gogan (1985) a résumé les douze fonctions majeures d'un tel bureau (tableau 3). Est-il nécessaire de dire que la recherche ne peut pas être gérée par un bureau de la recherche car celle-ci est une activité hautement individualisée? L'efficacité du bureau repose beaucoup sur la crédibilité de l'individu qui le dirige et sur les politiques incitatives de l'établissement. Parmi les politiques incitatives, mentionnons le dégrèvement de cours, l'octroi de fonds de démarrage de recherches et d'allocations de voyages sur une base très sélective.

Deuxièmement, une politique institutionnelle ferme sur la gestion des programmes est certainement un second facteur que nous, administrateurs et collègues professeurs, pouvons et devons mettre à profit et, je n'hésite pas à le dire, beaucoup mieux que dans les grandes universités. S'il existait une relation entre la gamme de cours offerts et la qualité de la formation de premier cycle, les grandes universités seraient beaucoup plus réputées qu'elles ne le sont à cet égard. 
TABLEAU 3

Fonctions d'un bureau de recherche

1. Élaboration des politiques.

2. Mise à jour et diffusion de l'information sur les sources de financement et les politiques des organismes subventionnaires.

3. Liaison avec les organismes de financement.

4. Centralisation des demandes et approbation au nom de I'université.

5. Élaboration et mise à jour d'un inventaire des recherches.

6. Aide aux groupes et aux particuliers dans la préparation des propositions.

7. Contrôle des rapports à soumettre.

8. Liaison avec les directeurs, doyens et toute autre personne, si nécessaire.

9. Négociation des contrats de recherche.

10. Contrôle adéquat des processus touchant les contrats, les soins aux animaux, etc.

11. Préparation des relations publiques pour les chercheurs.

12. Sondage d'opinion des membres de la communauté universitaire sur des sujets de recherches.

Or, tel n'est pas le cas! Pourquoi alors essayer de les imiter? Pourquoi devrions-nous être notre propre ennemi en multipliant les préparations de cours et les surcharges sous le faux prétexte d'offrir une formation comparable à celle des grandes universités. Oublions la fausse modestie et affirmons que notre modèle de formation au premier cycle est généralement supérieur à celui des grandes universités. 
Troisièmement, l'établissement, par l'entremise de son conseil de recherche, doit utiliser ses fonds internes de recherche de façon stratégique. Donnons deux exemples. Dans le domaine des sciences et du génie, il faut savoir faire la différence entre les chercheurs ayant perdu leur bonne réputation auprès du Conseil de recherches en sciences naturelles et en génie de ceux qui pourraient, avec un peu d'aide, redevenir allocataires de ressources. En conséquence, les subventions internes de recherches dans ce domaine doivent être plus sélectives et le niveau d'aide par projet plus élevé. Dans les sciences sociales et les humanités, les comités externes d'évaluation se basent beaucoup plus sur la valeur du projet que sur le dossier de publications du chercheur; dans cette optique, les fonds internes devraient encourager un plus grand nombre de projets avec un niveau d'aide financière moins élevé pour chacun. En résumé, les fonds internes doivent servir de palliatifs à ceux qui continuent à demander des subventions externes.

Qu'arrive-t-il aux chercheurs qui ne reçoivent ni subventions internes ni subventions externes? D'abord, certains affichent une excellente productivité de recherche sans recevoir aucun fonds en raison de leur domaine de recherche. D'autres forgent des alliances fort productives avec un ou des collègues plus nantis. Finalement, bon nombre de professeurs s'engagent dans des contrats de recherche. Afin d'éviter que les contrats de recherche deviennent purement une question d'argent pour le bénéficiaire du contrat, l'établissement devrait énoncer une politique très claire à cet égard. L'Université Laurentienne favorise les contrats de recherche reliés à ses centres de recherche qui, approuvés par le comité exécutif du centre en question, ont des retombées positives sur la production de recherche du professeur et qui, plus souvent qu'autrement, correspondent à la responsabilité d'une université en milieu régional. Dans un tel contexte, le danger de la course aux contrats de recherche pour des fins pécuniaires seulement est moins grand et le produit a plus de chances d'être considéré comme une activité légitime dans la structure de récompenses.

\section{CONCLUSION}

Dans l'ensemble, les petites universités constituent pour leurs régions des agents de développement socio-économique extrêmement importants et les grandes universités urbaines n'ont ni l'intétêt ni la compréhension nécessaires pour remplir ce rôle à leur place. En plus de continuer à orienter leurs activités sur un excellent enseignement de premier cycle, les petites universités sont beaucoup mieux placées, en raison de leur bureaucratie légère, pour faire des choix de recherche, d'abord restreints en nombres et ensuite axés davantage sur les réalités interdisciplinaires du milieu. Il ne fait aucun doute que ces choix profiteront davantage à la recherche appliquée au détriment de ce que certaines personnes des grandes universités se plaisent à appeler la recherche "lourde". Or, il est clair que la recherche "lourde" est fort coûteuse et élimine a priori un pourcentage élevé de professeurs-chercheurs si l'on en croit les données sur l'Université de Montréal 
(Bélanger et Lacroix, 1988). Sans écarter complètement ce type de recherche dans les petites universités, peut-être avons-nous plus de chances de répartir les efforts et les résultats de la recherche appliquée sur un plus grand nombre de professeurs et ceci en dépit de contraintes connues. Après tout, n'y-a-t-il pas un vent de décentralisation et un renouveau d'intérêt de la part de nos gouvernements en faveur des collectivités régionales?

\section{BIBLIOGRAPHIE}

Bélanger, C.H. et Lacroix, R. (1986). Measuring the effectiveness of research grant getting. $L a$ revue canadienne d'enseignement supérieur, 16 (1), 25-38.

Bélanger, C.H., Lacroix, R. et Raynauld, A. (1987). Les charges d'enseignement dans les universités québécoises : une réplique au Rapport Gobeil. La revue canadienne d'enseignement supérieur. 17 (3), 33-45.

Bélanger, C.H. et Lacroix, R. (1988). Faculty workload: who does the work?. Communication faite à la conférence annuelle de l'Association for Institutional Research, 314 Stone Building, Florida State University, Tallahassee, Florida 32306.

Conseil des Arts du Canada (1977). Besoins des chercheurs des petites universités. Rapport du groupe consultatif sur les besoins des chercheurs des petites universités, C.P. 1047, Ottawa, Ontario K1P 5V8.

Gogan, N. (1985). The development of research administration on management in Canadian universities. Conseil des sciences du Canada, Edifice Berger, 100, rue Metcalfe, Ottawa, Ontario K1P 5M1

Isaacs, C.F.W. (1976). Avoiding stagnation in small university departments. Rapport préparé par le Conseil des études supérieures de l'Ontario, Suite 839, 130, rue George, Toronto, Ontario M5S $2 \mathrm{~T} 4$.

Keller, G. (1983). Academic strategy: The management revolution in higher education. The Johns Hopkins University Press, Baltimore, Maryland.

Kerr, C. (1984). Presidents make a difference. Rapport de la Commission on Strengthening Presidential Leadership, Association of Governing Boards of Universities and Colleges, One Dupont Circle, Washington, D.C. 20036.

Lacroix, R. (1988). Le professeur d'université : Mythes et réalités. Présentation faite au Conseil des gouverneurs associés de l'Université de Montréal, C.P. 6128, Succursale A, Montréal, Québec H3C $3 \mathrm{~J} 7$

Oberlin College (1985). The Future of science at liberal arts colleges. Rapport d'une conférence tenue à Oberlin College, Ohio 44074.

Overgaard, H. (éd) (1974). Problèmes des chercheurs en sciences sociales dans les petites universités. Rapport d'un groupe de travail du Conseil de recherche en sciences humaines, 255, rue Albert, C.P. 1610, Ottawa, Ontario K1P 6G4.

Sibley, W.M. (1986) Strategic planning and management for change. La revue canadienne d'enseignement supérieur, 16 (2), 81-102. 


\section{UNIVERSITY ENTREPRENEURSHIP AND COMPETITION: THE CASE OF THE SMALL UNIVERSITIES}

\section{CHARLES H. BÉLANGER}

The image which Canada's fifty-odd universities present to the public is one which mirrors that of the country as a whole, namely a mosaic full of contrasts and enigmas. With $80 \%$ of their financing derived from public sources, they enjoy unequalled autonomy compared to other sectors of society. The independence that university professors enjoy is the envy of all those who are bound by rigid work schedules. All of the universities without exception assume the triple functions of teaching, research, and community service, but by and large, in the public perception, teaching is their one and only activity. Thanks to the collegial system of governance, they can call upon an immense fund of brainpower; but, by the same token, collegiality gives way to excessive individual hypersensitivity when it comes to goals, priorities, and institutional planning.

\section{THE SETTING}

Roughly half of Canada's universities have an enrollment of fewer than 5,000 students. One thinks mainly of such institutions as being regional in nature, geared mainly to undergraduate teaching, and, more often than not, drawn in the wake of the larger universities, these being associated with the large urban centres and geared to research. Even though it is only a question of emphasis rather than an either/or classification, this important distinguishing feature tends to make the coexistence of these two categories of universities resemble the division of a single country into two groups of people with differing cultures. On both sides there is a lack of appreciation of the strengths and weaknesses of the other group, which tends to make discussion more emotional than rational. The small universities say they are victims of the big university network when it comes to the allocation of research grants, while the large universities let it be understood that they are obliged to use double standards to accommodate the demands of the small universities. The facts, however, do not always support such allegations. The reports of the Social Sciences and Humanities Research Council show that the small universities receive grants at a slightly higher level than is warranted by the numbers engaged in research. According to the Natural Sciences and Engineering Research Council, the small universities are trailing behind, in terms of both successful applications and grant amounts. As for the question of double standards, it is interesting to note the results of a U.S. study: "The Future of Science at Liberal Arts Colleges" (Oberlin College, 1985). In general, the figures show that the 48 leading liberal arts colleges stand at the forefront, or just about, of all U.S. institutions of higher education, in the training of scientists (and that includes the multi-campus universities and the principal research centres). These data show that the colleges achieve their success largely through the close 
relationship established between professor and student. This same report not only points to an exceptionally high productivity rate for the leading liberal arts colleges, but it also shows them to be relatively cost-effective. Although no similar study has ever been done in Canada, there is a striking similarity between the American liberal arts college and the small university in Canada.

Competition. All universities nowadays, of whatever size, find themselves engaged in a competitive struggle, the consequences of which are beneficial for some and costly for others, depending on the mission they have selected for themselves and the leaders they have installed to pursue their particular paths of excellence. We have been told for the last two decades at least that higher education has become "big business", as though belonging to the private sector. Clearly, the business firm that cannot compete must of necessity close its doors. In his book entitled Academic Strategy (1983), Keller says that by 1995 between 10 and $30 \%$ of the 3,100 U.S. colleges and universities will have to close, as a result of lack of both students and resources. Since all our universities in Canada are financed by governments, the chances of their being shut down by the latter may be ruled out almost entirely. In the present circumstances, competition between institutions is only a pseudo-competition, for they always end up going to the government when they are in a tight spot. This will result, however, in a category of universities in very poor health. What can the small universities do to prevent this? Here is what Keller (1983) and Sibley (1986) advise:

1. The institution must have a President with the proven leadership qualities of a strategist and a general. If we are to accept the conclusions of Presidents Make a Difference (Kerr, 1984), it is becoming ever more difficult to attract university presidents who meet these requirements, because of the cumbersome decision-making processes and the hidden dangers that lie in wait for the unwary.

2. Our university enterprises will have to question themselves more closely and do some more rigorous self-examination as to how to manage change. What kind of business are we engaged in? What is our special role? What advantages do we possess as compared to other similar institutions? What goals should this university have attained ten years from now? What are our traditions, our values, our aspirations? How do we relate these traditions, values and aspirations to the continuously evolving world outside? What are our strengths and our weaknesses? In which corner of the market-place can we be sure of meeting the competition effectively? How shall we distinguish ourselves from the other players in the same league?

3. The notion of collegiality must change in two ways. Firstly, a spirit of cooperation between administrators and faculty will have to be established so as to promote the well-being of the enterprise. Secondly, we shall have to recognize the necessity of maintaining collegiality (meaning the collective 
control, by a group of peers, of matters within the scope of their expertise); but the responsibility for this collective control over everything which takes place in the institution will have to be restricted.

4. The concept of quality has in many instances remained just an idea; it must become a reality, translating into greater satisfaction for the student-consumer and in a more visible contribution to the community. This will necessarily bring about a more marked specialization and differentiation between institutions.

Academic entrepreneurship. Having defined in general terms the factors involved in institutional competition, let us now look at the professor as entrepreneur. Surprising as it may seem to some of us, says Lacroix (1988), a good analogy can be drawn between the entrepreneur in the business world and the university professor. "Both have a great degree of liberty in combining the resources at their disposal; both are judged essentially on their 'output'. Both must be inventive to succeed, and both are wholly responsible for their successes and failures. They are not there to carry out decisions made by others ..." The truth of the matter is that every professor is at once employee and entrepreneur: employee in the sense that he/she has to put in a certain number of hours, to conform to a certain time schedule, and to undertake a wide range of responsibilities toward the students and the department; he/she is also entrepreneur in the conduct of his/her own research activities.

The fact that the research function is the duty of every university professor, while the conduct of such research is left entirely to the dynamism and initiative of each professor, gives rise to extremely variable performances. A study (Bélanger and Lacroix, 1988) carried out at the University of Montreal, which is said to be a research university, revealed that $40 \%$ of those professors making up the most productive group in the University had to their credit:

- $91.1 \%$ of the highly specialized publications

- $73.7 \%$ of other external productions

- $63.6 \%$ of the completed Master's and Doctoral theses

- $65.4 \%$ of the grants and certain research contracts

- 12 teaching credits as against 12.4 for the faculty as a whole.

These results demonstrate that some have a highly developed spirit of enterprise while others have practically none. It is not clear to what extent these conclusions can be generalized. Insofar as the University of Montreal comes fifth in research productivity among the larger Canadian institutions (Bélanger and Lacroix, 1986), and in view of the fact that no such study has been conducted for the small universities, it is reasonable to assume that the same would be true of the $40 \%$ of high performance faculty members in the small universities. Even if the analogy were to prove accurate, however, it provides very little solace.

\section{THE CONSTRAINTS}

As competition for research grants from the three large federal funding councils heats up, the small universities realize that they are pitted against unequal forces, especially in territory coveted by research teams with established records. With 
Contribution of the top-ranking $40 \%$ of professors to total research output -- by discipline sector

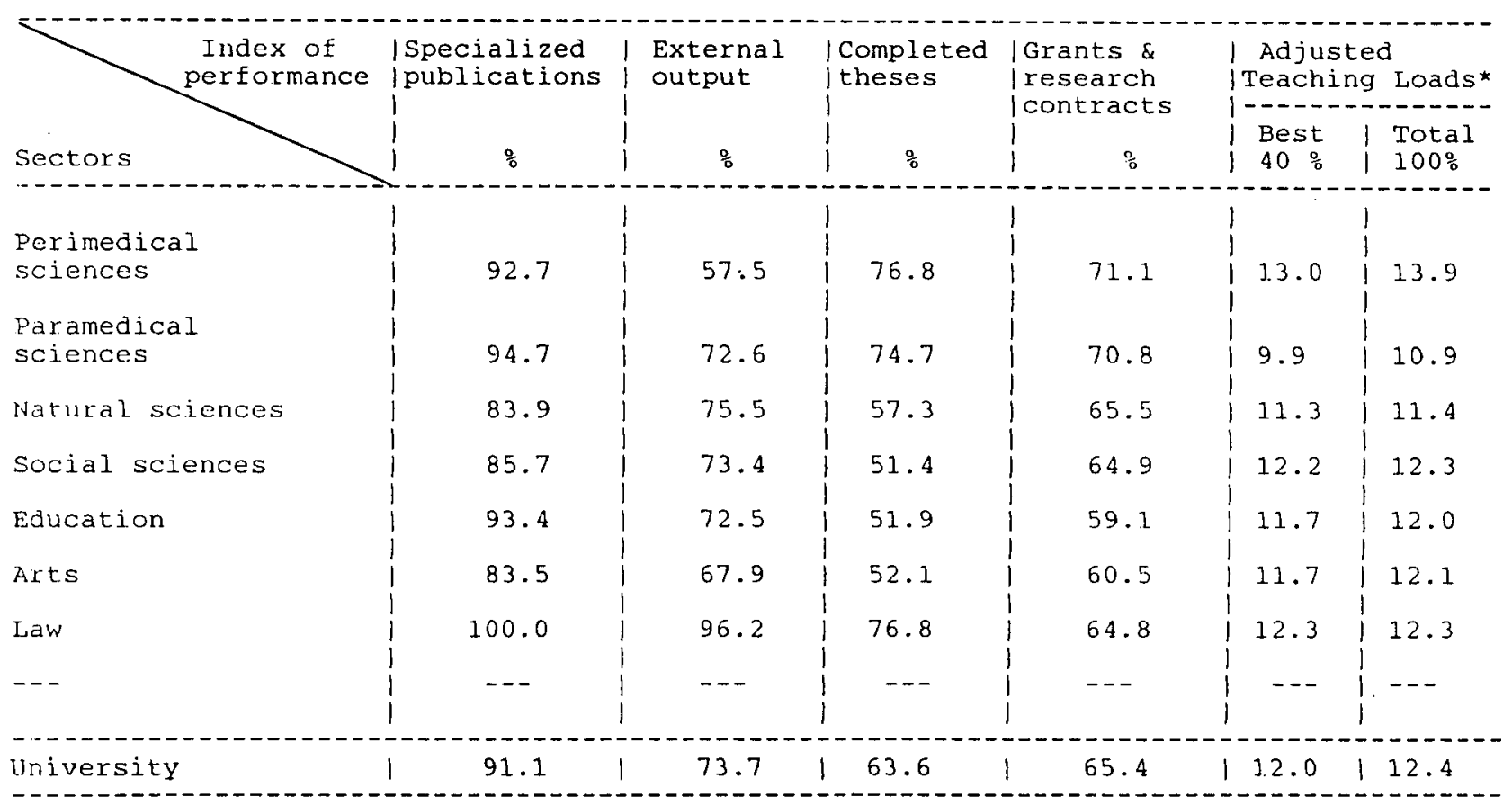

* Adjusted means that administrative/service work (e.g. chairmanship) was incorporated in tcaching loads as per departmental/institutional practices. 
regard to grants to individual researchers, the problem is of a different sort. It is just this intense competition that made the small universities organize an annual conference on research, beginning in 1985, for the purpose of discussing their problems and looking at certain strategies. It appears that after three important reports on these matters were published in the mid-seventies (Overgaard, 1974; Isaacs, 1976; Arts Council, 1977), interest fell off in subsequent years. And yet, it is not really surprising to discover that the constraints which surrounded the small universities ten years ago have not changed very much today. We shall review briefly the most important of these constraints, not for the pleasure of making excuses, but rather in order to recognize more clearly the limitations of the small university and to formulate a plan of attack, as would the head of a small business.

1. Teaching loads. A study of 39 Canadian universities carried out by Bélanger, Lacroix and Raynauld (1987) shows that in fact teaching loads in the small universities, excluding the Province of Quebec, are about 17-17.5 credits per year, compared to 13-13.5 credits for the large universities, i.e., a difference of almost $25 \%$. The same study revealed that the faculty divided their energies in different proportions between teaching and research in the two types of universities (See Table 2). In general, it may be said that the smaller the institution, the greater the teaching load and the smaller the class-size - which lends credence to the argument put forth by the large universities.

2. Lack of Graduate Programmes. There is no question but that the small universities have always had difficulty in creating and sustaining a critical mass of post-graduate studies. The small universities argue that they do not have the advantage of the services of graduate students as teaching and research assistants. On the other hand, the large universities put forward the counter argument that the responsibility for young scholars engaged in M.A. and Ph.D. programmes of study makes much greater demands on the professor's time and attention than is the case for undergraduate students. The graduate student/ professor ratio is less than 0.5 for the small universities, and approximately 2.0 for the large universities (Bélanger, Lacroix and Raynauld, 1987).

3. Size of teaching units. This appears to be one of the most important questions to be addressed. Some U.S. studies have suggested that the critical number of professors per department may vary according to the discipline, but that on the whole it is difficult to reduce department size to below ten or thereabouts. In actual fact, most units representing disciplines in the small universities number fewer than the threshold figure, and this has some rather negative consequences. Any one professor in a small university has to prepare a greater variety of courses. It is practically impossible to put together research teams within a particular discipline; for example, a small university may have the services of one specialist in Analytical Chemistry, whereas there may quite often be as many as six or eight in a large university. The small universities face the inherent danger of isolation within a particular discipline, not to speak of the geographical isolation which separates one from colleagues in other institutions. Conflicts due to differences of personality and differences of approach seem to be more pronounced and more personal. It is much more 
TABLE 2

Apportionment of energies on the part of faculty members

\begin{tabular}{|lccc|}
\hline & $\begin{array}{c}\text { Small } \\
\text { universities }\end{array}$ & $\begin{array}{c}\text { Large } \\
\text { universities }\end{array}$ & Weighted \\
Functions & $(\mathrm{N}=16)$ & $(\mathrm{N}=14)$ & $(\mathrm{N}=31) *$ \\
\hline \hline Teaching & $54 \%$ & $48 \%$ & $52 \%$ \\
Research & $26 \%$ & $33 \%$ & $28 \%$ \\
Service & $20 \%$ & $19 \%$ & $20 \%$ \\
\hline
\end{tabular}

* The total of 31 does not include the 8 quebec universities which responded.

difficult to "cover up" one or two colleagues in a team of five or six, than to do the same for eight or nine in a team of thirty-five, when the time comes to be evaluated for the purpose of introducing or renewing graduate programmes. Lastly, the small unit often lacks a spirit of competition and challenge.

4. The dearth of "models". In management, it is said, "If you want to go places, associate with movers". The same can be said of research. Young professors need to be in proximity to experienced research professors, and even to become associated with them, in order to learn the inner workings of the entrepreneurial aspect of the research function. Some outstanding corporations and some highly regarded academic departments provide excellent models for emulation. There are researchers in the small universities who can and do perform this function, but they are few and far between. In the first place, it is becoming harder to attract them: the difficulty can be attributed to heavier teaching loads and to the dearth of graduate programmes. Moreover, the demands made on these professors are already excessive, by virtue of their general excellence. Over the years they have lessened the pace of their research activities, because they have had to accomplish tasks that no-one else would or could assume.

5. Inadequate infrastructure and resources. Obviously the notion of infrastructure and resources is never absent from the minds of researchers in both the small and the large universities. This notion takes on importance and a different character according to whether you belong to a laboratory discipline like Chemistry, or a pencil and book discipline like Literature. Considering the three large federal funding councils, one discovers that the average grant for a professor lies between $\$ 14,000$ and $\$ 17,000$ in the research universities, depending on whether the institution offers doctoral programmes, and 
especially programmes in the Health Sciences which qualify for grants from the Canada Medical Research Council. This important variation between the two types of universities is, of course, no answer to the shortage of equipment, infrastructure and research support staff in the small universities.

6. The stigma of the small university. Right from the start the small universities find themselves with two strikes against them, as a result of their historical context, their emphasis on the student-teacher relationship, their isolation from the large population centres, and the simple fact that they are small in size. They therefore constitute a narrower, less visible network of professors, as well as a network of graduates who are limited to certain disciplines and professions, and not always overly represented in the decision-making apparatus of our organizations.

\section{STRATEGIC PLANNING}

If the enumeration of all these constraints discourages you, I suggest to you that you forsake the world of the small university, because research cannot be separated from teaching without in the long run constituting a dead-weight for the students and the institution as a whole. If, on the other hand, you are competitive and aggressive, a requirement sine qua non of any entrepreneur, let us examine together some ways to rise above these constraints.

Stage one. Firstly, we have to go back to the first point made in talking about strategic planning. The institution should provide itself with a President, a strategist who understands not only the notion of the university as an enterprise, but above all has her/his idea of this university as an enterprise. The presence of this key figure is so fundamental as to set in motion a series of decisions that will assuredly revitalize the institution. This new strategist generally heads up a dynamic team of aides sharing their leader's vision for this institution.

Secondly, the administration - right down to the departmental chairman - must understand, and explain to all concerned, that hiring, promotion and tenure decisions define research policies. If the institution hires and keeps the sort of faculty who have neither the ability nor the will to become the head of a small business, then frustration will overtake the entrepreneurial potential.

In the third place, the question arises as to which few lines of research the institution shall give preference. Such choices, though hard to make, are essential for the economical allocation of resources and critical mass between disciplines. Just as discipline-oriented team research is the bastion of the large universities, particularly with respect to the Natural Science and Engineering Research Council and the Medical Research Council, so the small universities should make interdisciplinary research their trademark and their banner. If research is organized around a limited number of institutional axes or centres, this will have positive results for the independent professor-researcher: it will narrow down institutional priorities and provide a focal point for the exchange of ideas with other 
TABLE 3

Functions of a research office

\begin{tabular}{|l|} 
1. Policy planning. \\
3. Revise and distribute information on financing \\
sources and on the policies of foundations. \\
4. Centralize applications and approve them \\
on behalf of the university. \\
5. Establish and maintain an inventory \\
of ongoing research \\
6. Assist groups and individuals in the \\
preparation of proposals. \\
7. See that reports are duly submitted. \\
9. Mintain liaison with departmental heads, \\
10. Exercise due control of procedures relating to \\
contracts, care of animals, etc. \\
11. Prepare public relations materials for researchers. \\
12. Conduct opinion surveys within the university \\
community on topics for research.
\end{tabular}

similarly-minded researchers; moreover, the latter retain the freedom to pursue their own personal research interests if they so desire.

Stage two. It should be pointed out right away that the second stage will be effective only if the three conditions mentioned in the first stage are clearly established. From now on it is a question of instrumentation, means and policies which the institution will introduce in order to foster a climate of research and to support the efforts of the researchers. In the first place, it seems that here an effective research office plays a crucial role. In his report to the Science Council of Canada, Gogan (1985) summed up the twelve main functions of such a body (Table 3). We scarcely need to be reminded that research, being a highly individual activity, cannot be managed out of a research office. The effectiveness of such an office is to a great extent determined by the credibility of its director, and by institutional policies with respect to incentives. These might include course reductions, allocation of start-up research funds and travel grants on a very selective basis. 
Secondly, a firm institutional policy on the management of programmes is certainly a factor that administrators and faculty colleagues can and must put to good use, and I have no hesitation in saying, much better use than in the large universities. If there were any relation between the abundance of courses offered and the quality of undergraduate teaching, the large universities would have a much greater reputation than they now enjoy in this respect. Since this is so, there is no reason at all for us to try to copy them: why should we act as our own worst enemy by increasing the number of course preparations and overloads on the false pretext of offering an education comparable to that of the large universities? Let us throw off any false modesty and say outright that our undergraduate teaching model is generally superior to that of the large universities.

Thirdly, the institution, through its research council, should utilize its internal research funds strategically. Let us take two examples. In the area of Science and Engineering, we have to make a distinction between those researchers who have lost their rating with the Natural Science, and Engineering Research Council and those who, with a little assistance, could once more become eligible. Consequently, our internal research grants in this area have to be more selective and the level of assistance higher. In the Social Sciences and Humanities, the external evaluation committees base their decisions much more on the value of the project than on the publication record of the researcher; in consequence of this, the internal funds should serve to encourage a greater number of projects with a lower level of financial assistance for each one. In short, internal funds must serve as palliatives for those who continue to look for external grants.

What happens to those who receive neither internal nor external funding? Note first that some of them show excellent research productivity, but, because of their research field, they are denied funding. Others form very fruitful partnerships with one or two colleagues who are better off. Lastly, many professors sign research contracts. To prevent research contracts from becoming purely a question of income for the contracting party, the institution should set forth clear guidelines in the matter. Laurentian University favours research contracts which are linked to research centres, are approved by the executive committee of the centre in question, have a positive effect on the research productivity of the individual scholar, and, more often than not, coincide with the responsibility such a university has to its regional environment. In this way, the danger of the race to acquire research contracts for financial gain alone is reduced, and the resulting product stands a greater chance of being viewed as a legitimate activity against the overall pattern of rewards.

\section{CONCLUSION}

On the whole, the small universities are extremely important agents of socioeconomic change for their regions, and the large urban universities have neither the interest nor the understanding required to play this role in their place. In addition to continuing to steer their activities in the direction of excellence in 
undergraduate teaching, the small universities are much better placed, by reason for their lighter administrative superstructure, to make research choices that are not only limited in number but also geared to the interdisciplinary realities of their environment. There is no doubt that these choices will favour applied research, to the detriment of what some members of the large universities like to call "heavy" research. As it turns out, "heavy" research is very expensive and eliminates on principle a goodly percentage of professor-researchers, if one accepts the data for the University of Montreal (Bélanger and Lacroix, 1988). Without setting aside completely this type of research in the small universities, perhaps we have a chance to spread our applied research efforts and results over a greater number of professors, and this in spite of known constraints. After all, are we not witnessing a move toward decentralization, and a renewal of preoccupation, on the part of our governments, with regional collectivities?

\section{BIBLIOGRAPHY}

Bélanger, C.H. \& Lacroix, R. (1986). Measuring the effectiveness of research grant getting. Canadian Journal of Higher Education, 16 (1), 25-38.

Bélanger, C.H., Lacroix, R. and Raynauld, A. (1987). Les charges d'enseignement dans les universités québécoises: une réplique au Rapport Gobeil. Canadian Journal of Higher Education, 17 (3), 33-45.

Bélanger, C.H. \& Lacroix, R. (1988). Faculty workload: who does the work? Presented at the Annual Meeting of the Association for Institutional Research, Phoenix, Arizona.

Canada Council (1977). Needs of scholars at small universities. Report of the Consultative Group on the Needs of Scholars at Small Universities, Ottawa.

Gogan, N. (1985). The development of research administration on management in Canadian universities, Science Council of Canada, Ottawa.

Isaacs, C.F.W. (1976). Avoiding stagnation in small university departments. Report prepared by the Ontario Council on Graduate Studies, Toronto.

Keller, G. (1983). Academic strategy: The management revolution in higher education. The Johns Hopkins University Press, Baltimore.

Kerr, C. (1984). Presidents make a difference. Report of the Commission on Strengthening Presidential Leadership, Association of Governing Boards of Universities and Colleges, Washington, D.C.

Lacroix, R. (1988). Le professeur d'université: Mythes et réalités. Presented at the Conseil des gouverneurs associés of the Université de Montréal.

Oberlin College (1985). The future of science at liberal arts colleges. Report of a conference held at Oberlin College, Ohio.

Overgaard, H. (ed.) (1974). Problèmes des chercheurs en sciences sociales dans les petites universités. Report of a task force of the Social Sciences and Humanities Research Council, Montreal.

Sibley, W.M. (1986). Strategic planning and management for change. Canadian Journal of Higher Education, $16(2), 81-102$. 